\title{
The Moderating Effect of Store Category on the Relationship Between Corporate Social Responsibility and Customer Buying Intention: An Abstract
}

\author{
Zhi Pei, Ruiliang Yan, and Chris Myers
}

\begin{abstract}
In this study, CSR is categorized into five domains: community support, employee support, product, environment, non-domestic operations (Sen and Bhattacharya 2001). To have a comprehensive understanding of CSR, each domain is measured and examined the relationship with customer buying intention. While the extant literature examines CSR broadly and suggest it has no influence on customer purchase behavior, our research argues that the conceptualized domains of CSR do influence customer buying intention, especially when the specific domain of CSR activities relates to customer self-interests and fits the store category (e.g., convenient store, drug store, apparel store, supermarket).

Toward this goal, we conducted 50 face to face Qualitative interviews to get indepth understanding of customers' attitudes and behaviors toward conceptualized domains of CSR. The content analysis shows that if there is a fit between store and its CSR activity (e.g., CVS pharmacy stopped selling tobacco), customers are more likely to have positive purchase intention. To confirm the Qualitative findings, we will further collect quantitative data using survey based method. Data will be analyzed using Structure Equation Modeling (SEM). Using a comprehensive conceptualization of CSR, this study will fill the gap in the current CSR literature and have great managerial contribution as well.
\end{abstract}

Z. Pei $(\bowtie) \bullet$ R. Yan $\bullet$ C. Myers

Texas A\&M University-Commerce, Commerce, TX, USA

e-mail: Zhi.Pei@tamuc.edu; Ruiliang.Yan@tamuc.edu; Chris.Myers@tamuc.edu 